\title{
Cipango Cipango
}

Cahiers d'études japonaises

\section{$24 \mid 2021$}

Eugénisme dans le Japon moderne et contemporain

\section{Aoi shiba no kai : Un mouvement de personnes handicapées face à l'eugénisme}

Aoi shiba no kai: a group of disabled people fighting eugenics

\section{Anne-Lise Mithout}

\section{OpenEdition}

1 Journals

\section{Édition électronique}

URL : https://journals.openedition.org/cipango/4583

DOI : $10.4000 /$ cipango.4583

ISSN : 2260-7706

Éditeur

INALCO

\section{Édition imprimée}

Date de publication : 20 octobre 2021

Pagination : 129-162

ISBN : 9782858313969

ISSN : 1164-5857

\section{Référence électronique}

Anne-Lise Mithout, « Aoi shiba no kai : Un mouvement de personnes handicapées face à

l'eugénisme », Cipango [En ligne], 24 | 2021, mis en ligne le 11 octobre 2021, consulté le 09 avril 2022.

URL : http://journals.openedition.org/cipango/4583 ; DOI : https://doi.org/10.4000/cipango.4583

\section{(c) (i) (9)}

Cipango est mis à disposition selon les termes de la Licence Creative Commons Attribution - Pas d'Utilisation Commerciale 4.0 International. 


\title{
Aoi shiba no kai : Un mouvement de personnes handicapées face à l'eugénisme
}

Aoi shiba no kai: a group of disabled people fighting eugenics

\author{
Anne-Lise Mithout \\ Université de Paris - CRCAO
}

Haha yo! Korosuna母よ殺すな 《Maman! Ne me tue pas » fut l'un des slogans les plus marquants et les plus controversés lancés par l'association Aoi shiba no kai 青い芝の会, pionnière du mouvement d'émancipation des personnes handicapées au Japon au début des années 1970. Il exprime toute la violence de la contestation par celles-ci d'un ensemble de politiques, d'actions, de pensées et de représentations sociales désigné sous le nom d'《eugénisme » (yūseigaku 優 生学).

Dans le Japon de l'immédiat après-guerre, il existe déjà des associations civiles de personnes handicapées, nées des cendres des mouvements d'avantguerre. Chacune de ces associations exprime des revendications spécifiques à une catégorie de handicap (invalides de guerre, personnes aveugles, malades de la tuberculose...). Dès leur création, elles font valoir certaines idées qui deviendront plus tard communes à tous les mouvements de personnes handicapées (sortie du milieu hospitalier, accès à l'emploi). Mais elles ne visent pas à présenter un front uni des « personnes handicapées ». C'est Aoi shiba no kai qui va jouer ce rôle fédérateur. 
Dès 1947, d'anciens élèves d'écoles spécialisées de Tōkyō, atteints de paralysie cérébrale, créent un journal, Shinonome しののめ destiné à favoriser les échanges entre pairs vivant avec ce handicap. Ce sont ces échanges, devenant au fil des années plus théoriques et politiques, qui inspirent à une partie des contributeurs (tous anciens élèves de l'école spécialisée Kōmei, dans l'arrondissement de Setagaya à Tōkyō) la volonté de créer, en 1957, l'association Aoi shiba no kai ${ }^{1}$ avec l'objectif de promouvoir la mise en pratique des idées défendues dans le journal. L'association est initialement composée de quarante membres, tous atteints de paralysie cérébrale, et vise à favoriser non seulement les échanges d'opinion entre ses adhérents (notamment par le biais de publications écrites), mais aussi l'organisation d'activités de loisirs et la correspondance avec des associations américaines de personnes ayant une paralysie cérébrale ${ }^{2}$. En l'espace de dix ans, des branches de l'association se créent dans tout le pays, et l'activité se politise : l'association soulève des questions liées au traitement du handicap dans la société, en particulier à la protection sociale, et tente d'entreprendre des négociations avec le ministère de Santé et de la Protection sociale (Köseishō, 厚生省). En 1964, l'association met en place une expérience de vie communautaire dans les montagnes du département d'Ibaraki (le « village Maharaba », Maharaba mura $\checkmark$ ハラバ村), encadrée par Osaragi Akira 大仏空 (1930-1984), prêtre bouddhiste de la secte Jōdo shinshü ${ }^{3}$. Dans le même temps, elle s'organise au niveau national en créant la Fédération nationale Aoi shiba no kai (Zenkoku Aoi shiba no kai

1. Ce nom qui signifie «Association de l'herbe verte », a été choisi par les membres pour véhiculer l'idée que «l'herbe reste, même si elle est piétinée à de nombreuses reprises », un message symbolisant la capacité de résistance des personnes handicapées.

2. Correspondance dont le volume demeure cependant limité dans les premières années, du fait que peu de membres de l'association maitrisent l'anglais. Les échanges internationaux d'Aoi shiba no kai ne prendront leur essor qu'à la fin des années 1970 / au début des années 1980 lorsque, à l'occasion de la préparation de l'Année internationale des personnes handicapées (1981) organisée par l'ONU, de grandes entreprises mettent en place des bourses permettant à des militants handicapés de partir séjourner plusieurs mois aux États-Unis. C'est le cas notamment de la militante féministe Asaka Yūho qui participe à un programme sponsorisé par l'entreprise Mister Donuts, comme elle le raconte dans son autobiographie (AsAKA, 1993).

3. Osaragi a hérité de son père la charge du temple Kanjōji (願成寺), mais il a également étudié dans sa jeunesse dans un séminaire catholique. Il est également marxiste. 
sōrengōkai 全国青い芝の会総連合会) et entreprend des actions destinées à faire entendre au grand public la voix des personnes handicapées ${ }^{4}$ : manifestations, distribution de tracts, rencontres avec d'autres associations citoyennes, réalisation d'un film documentaire ${ }^{5}$...

Si le discours d'Aoi shiba no kai s'appuie sur des idées déjà énoncées par les autres associations de personnes handicapées (notamment la volonté de dé-médicalisation du handicap), il se distingue néanmoins par la radicalité de sa contestation de l'ordre social établi. C'est en particulier Aoi shiba no kai qui émet pour la première fois une revendication identitaire liées au handicap (celle $\mathrm{du} \ll$ droit d'exister » en tant que personne handicapée), et non plus seulement des demandes de soutien matériel (accordé dans une logique qui demeure le plus souvent celle de la charité). L'association prône ainsi le passage d'une logique de compensation du handicap (qui demeure perçu comme un désavantage, un manque, un défaut) à une logique de reconnaissance (dans laquelle on ne chercherait plus à « masquer » le handicap mais à le prendre en compte comme une situation spécifique).

L'un des principaux chantres de ce changement est Yokozuka Kōichi 横塚晃 - (1935-1978), membre du village Maharaba puis vice-président de la branche de Kanagawa d'Aoi shiba no kai, et enfin président de la fédération nationale de 1973 jusqu’à sa mort en 1978. Ses écrits sont regroupés dans un recueil intitulé Haha yo! Korosu na (母よ! 殺すな Maman! Ne me tue pas) ${ }^{6}$, paru pour la première fois en 1975 puis, réédité en 2007 dans une édition augmentée préfacée par le sociologue Tateiwa Shin.ya.

Le concept d'eugénisme est au cœur de l'argumentation politique de Yokozuka qui l'utilise pour dénoncer la situation dans laquelle vivent les personnes

4. Si cet article porte uniquement sur le début des années 1970, période charnière à laquelle le discours d'Aoi shiba no kai émerge brusquement sur la scène politique et soulève de vives réactions, mentionnons que l'association existe toujours à l'heure actuelle et continue son travail militant, quoique celui-ci soulève maintenant moins de polémique qu'à ses débuts.

5. Sayōnara CP. Le film n'est pas réalisé par les membres d'Aoi shiba no kai eux-mêmes, mais par le réalisateur Hara Kazuo (原一男), dont c'est le premier film. Voir HarA, 2009.

6. Yокоzuka, 2007. 
132 Cahiers d'études japonaises nº 24

handicapées au Japon et faire entendre la voix de celles-ci sur la scène politique, en particulier dans le contexte du projet de révision de la loi de protection eugénique (Yūsei hogo-hō 優生保護法). Cette dénonciation s'appuie sur une définition quelque peu floue de l'eugénisme, rassemblant sous ce nom un vaste ensemble de situations diverses, au sujet desquelles l'analogie abondamment utilisée avec l'eugénisme nazi peut parfois sembler dérangeante. C'est pourquoi ce discours a suscité des réactions virulentes. Néanmoins, aussi provoquante que puisse parfois apparaître la rhétorique anti-eugénique de Yokozuka, elle est à l'origine de transformations majeures dans la perception sociale des personnes handicapées au Japon et, plus encore, dans la perception par celles-ci de leur propre identité.

Comment, et avec quelles conséquences, Yokozuka Kōichi fait-il de la lutte contre « l'eugénisme » un instrument d'émancipation et d'affirmation de soi des personnes handicapées?

Cet article s'appuie sur les écrits de Yokozuka pour en dégager les principaux arguments et montrer par quel mouvement de pensée il associe au contexte de politique eugéniste la nécessité d'une revendication identitaire liée au handicap. Pour ce faire, il nous a semblé nécessaire de citer et traduire de larges extraits des écrits de Yokozuka, pour en restituer la virulence (et parfois, les excès), car c'est cela même qui en fait la grande originalité : sa radicalité dans la manière d'affirmer non seulement le «droit à l'existence » des personnes handicapées, mais le handicap lui-même, comme situation particulière devant faire l'objet d'une reconnaissance publique et non être vécue individuellement comme une honte.

Il est essentiel de mentionner que la « voix des personnes handicapées » présentées ici est en réalité celle des personnes en situation de paralysie cérébrale, puisque ce sont elles qui composent Aoi shiba no kai. Yokozuka écrit le plus souvent au nom des 《 personnes handicapées 》 (shōgaisha 障害者), mais ne cherche pas à donner de définition du « handicap » (shōgai, 障害), ni à circonscrire l'ensemble des situations recouvertes par ce terme. Du fait qu'il met en avant la situation de discrimination que subissent les personnes handicapées dans la société, et non pas les difficultés matérielles liées à une déficience médicalement identifiée, il préfigure d'une certaine façon les penseurs du « modèle social du handicap ${ }^{7} »$,

7. Cette expression est néanmoins anachronique concernant Yokozuka : le « modèle social du handicap » naîtra dans les pays anglo-saxons dans les années 1980 et ne sera repris par les sociologues japonais qu’à partir du début des années 2000. 
c'est-à-dire la définition du handicap en tant que « désavantage social » plutôt que « déficience médicale ». Son discours se veut donc généraliste. Les revendications d'émancipation qu'il avance seront d'ailleurs suivies d'effet pour un ensemble de « personnes handicapées » beaucoup plus large que les personnes ayant une paralysie cérébrale ${ }^{8}$. Néanmoins, la spécificité de son point de vue doit être soulignée, en ce sens qu'il n'est pas certain que celui-ci soit parfaitement représentatif de la situation de toutes les personnes handicapées ${ }^{9}$; l'accueil de ce point de vue par une partie de la population en situation de handicap sera d'ailleurs parfois mitigé ${ }^{10}$.

Cet article analyse comment Yokozuka fait de la lutte anti-eugénique l'argument central de son discours d'émancipation. On cherche à montrer que « l'eugénisme » dénoncé par Yokozuka est, autant qu'une réalité historique, un concept-repoussoir permettant d'avancer une argumentation politique. Le discours anti-eugénique, parfois provocant, vise à favoriser l'affirmation identitaire des personnes handicapées (en tant que personnes handicapées, contre une société normalisatrice, contre un eugénisme latent) et leur émancipation (hors des établissements spécialisés, hors des contraintes normatives imposées par la société). Cependant, cette affirmation se fait au prix de vives réactions, aboutissant notamment à des divergences avec d'autres mouvements de contestation sociale qui, bien que travaillant sur des enjeux proches, se voient placés par ce discours

8. En témoigne notamment Iyashi no sekushī torippu, le récit autobiographique d'Asaka Yūho (AsAKA, 1993), activiste féministe atteinte de malformations osseuses ayant fait ses premiers pas militants avec Aoi shiba no kai, qui relate la révélation identitaire qu'a été pour elle la découverte du discours d'affirmation de soi défendu par l'association. Voir aussi : Mithout, 2018.

9. Pour reprendre l'anachronisme précédent, l'une des critiques qui sera adressée ultérieurement au «modèle social du handicap » est qu'il se fonde avant tout sur l'expérience des personnes en situation de handicap physique et ne rend pas compte de la diversité des « situations de handicap », en particulier des expériences subjectives liées aux handicaps mentaux. Pour une présentation détaillée du « modèle social du handicap » et de ses critiques, voir SHAKESPEARE, 2006.

10. Yoкоzuka, 2007, p. 97 (article original : Yokozuka Kōichi, « Aru shōgaisha undō no mezasu mono ある障害者運動の目指すもの [De certaines visées du mouvement des personnes handicapées] », Jurisuto ジュリスト, édition spéciale, octobre 1974). 
dans le camp des «oppresseurs » (notamment les associations féministes et les associations de parents).

\section{Yokozuka Kōichi : une quête d'émancipation marquée par la question de l'eugénisme}

Les années 1960-1970 ont été marquées dans de nombreux pays par des mouvements d'émancipation des personnes en situation de handicap, en particulier sous l'influence des associations anglo-saxonnes. Aux États-Unis, le Disability Right Movement est né dans les années 1960, dans le prolongement du mouvement pour les droits civiques et du mouvement féministe. Il aboutit au développement de recherches mettant en lumière les barrières auxquelles les personnes handicapées doivent faire face dans leur vie quotidienne (notamment pour se déplacer dans l'espace public) et les solutions possibles pour y remédier. De ce mouvement naît l'Independent Living Movement, qui émerge en Californie en 1970. Independent Living défend l'idée que les personnes en situation de handicap doivent pouvoir vivre au sein de la société et jouir des mêmes droits que leurs concitoyens. Le mouvement s'appuie sur l'idée que les personnes handicapées elles-mêmes sont les meilleurs experts du handicap et doivent avoir le droit de déterminer par ellesmêmes ce qui est bon pour elles, sans que leur parole soit confisquée (notamment par les médecins).

Si le mouvement japonais est indéniablement inspiré par les mouvements internationaux, comme en témoigne l'utilisation récurrente dans les revendications de l'expression jiritsu seikatsu (自立生活, vie en autonomie, traduction d'independent living), il porte néanmoins des revendications propres à la société japonaise, ancrées dans les enjeux sociaux et politiques d'après-guerre et marquées par des différences significatives avec les pays anglo-saxons ${ }^{11}$. La principale est la place accordée à l'idée d'une lutte contre « l'eugénisme » (yüseigaku 優生学) ou la « pensée eugénique » (yüsei shisōo 優生思想), beaucoup plus présente qu'aux États-Unis ou en Angleterre.

11. Sugino, 2007, p. 221. 
Sans revenir sur l'histoire de l'eugénisme au Japon ${ }^{12}$, il est essentiel de mentionner que le texte fondateur de la politique eugénique d'après-guerre, la loi de protection eugénique (1948), s'ouvre de la manière suivante :

Article 1 (objectif de cette loi)

Cette loi a pour objectif à la fois de prévenir la naissance d'une descendance mauvaise d'un point de vue eugénique et de protéger la vie et la santé des mères.

第一条【この法律の目的】

この法律は、優生上の見地から不良な子孫の出生を防

止するとともに、母性の生命健康を保護することを目的 とする。

Cette expression, « une mauvaise descendance » (furyō na shison 不良な 子孫), est la clé du discours anti-eugéniste de Yokozuka. C'est elle qui fonde la volonté de celui-ci de défendre le droit à l'existence des personnes handicapées et, dans une tentative de réappropriation du stigmate, l'affirmation identitaire du handicap revendiquée par Aoi shiba no kai.

Avant d'entrer dans l'analyse des écrits de Yokozuka, revenons sur le parcours de celui-ci ${ }^{13}$. Yokozuka est né dans le département de Saitama en 1935 et a contracté une paralysie cérébrale à l'âge de 10 mois à la suite d'une forte fièvre : il est presque entièrement paralysé et a de lourdes difficultés d'élocutions. Il connaît le destin classique des personnes handicapées à cette période, c'est-à-dire qu'il vit chez ses parents, entièrement pris en charge par sa famille, jusqu'à ses seize ans, puis est admis dans un établissement spécialisé pour recevoir une éducation correspondant au niveau de l'école primaire, puis du collège qu'il est obligé de quitter à 25 ans en raison de la limite d'âge en vigueur dans les établissements. Il vit alors un an en établissement spécialisé puis parvient à s'installer à son propre domicile. À 28 ans, en 1964, il part s'installer dans le village Maharaba fondé par

12. Voir à ce sujet Thomann, 2015 ; Matsubara, 2000.

13. Voir Yokozuka, 2007, p. 105-118 (article original : « Aru shōgaisha undō no mezasu » ; ainsi que SUzuki, 2010 ; Yoritaka, 2012, p. 48-77. 
Aoi shiba no kai sous l'égide du prêtre bouddhiste Osaragi Akira. Il vit alors en communauté avec d'autres personnes ayant une paralysie cérébrale, dans un milieu non médicalisé, en autonomie et presque en autarcie. Des couples se forment au sein de la communauté, des enfants naissent, et c'est à la suite de ces naissances que la communauté se disloque, certains couples choisissant de « revenir dans la société valide » pour permettre à leurs enfants (valides) de bénéficier d'une vie ordinaire. Yokozuka est l'un des derniers à quitter la communauté mais retourne finalement vivre avec son épouse dans le département de Kanagawa. Cette expérience le marque profondément, à la fois parce qu'elle est la preuve que des personnes ayant une paralysie cérébrale peuvent vivre de manière indépendante dans un milieu non médicalisé et parce que l'influence d'Osaragi Akira et du bouddhisme de la Terre Pure ${ }^{14}$ va laisser une empreinte durable sur sa pensée ${ }^{15}$.

Le discours d'émancipation et d'affirmation de soi de Yokozuka peut se décomposer en trois étapes : la volonté de prendre la parole « du point de vue des assassinés », de ceux que la société eugénique opprime et parfois supprime, la dénonciation d'un « eugénisme intériorisé » au cœur du fonctionnement de la société, issu en particulier des normes industrielles, et l'appel à une affirmation identitaire forte comme seule réponse aux pressions que cet eugénisme fait peser sur l'existence des personnes handicapées.

14.À l'inverse, les sources d'inspiration de Yokozuka en matière de politique sont moins claires. Osaragi Akira est marxiste, et le mouvement des personnes handicapées est plutôt marqué politiquement à gauche. Mais les écrits de Yokozuka ne font pas explicitement référence à une pensée/un penseur politique précis, ni à un courant, même si sa dénonciation de la société productiviste apparaît comme une forme d'anti-capitalisme. Rappelons que le handicap, à cette période, ne fait pas l'objet d'un positionnement clair des partis. C'est pourquoi Aoi shiba no kai, afin d'augmenter la visibilité politique des enjeux liés au handicap, entreprendra des négociations avec tous les partis sans s'associer spécifiquement à aucun. L'association cherche en particulier à dialoguer avec le Parti libéral-démocrate qui est alors au pouvoir, afin de faire entendre son point de vue sur les questions de protection sociale.

15. Voir Yokozuka, 2007, p. 105-118 (article original : « Aru shōgaisha undō no mezasu mono ») ; Yoritaka, 2012 


\section{Un discours « du point de vue des assassinés »}

L'évènement déclencheur de l'action politique d'Aoi shiba no kai est une affaire judiciaire survenue à Yokohama en 1970. Une mère tue volontairement sa fille de deux ans, atteinte de paralysie cérébrale et, à l'ouverture de l'enquête, une association de soutien à la mère se mobilise pour demander la clémence des autorités en mettant en avant le fait qu'il s'agissait d'un acte de désespoir lié à l'impossibilité de trouver un établissement spécialisé pour accueillir l'enfant. L'enquête s'oriente alors sur la question de savoir s'il existe réellement un manque d'établissements spécialisés dans la ville et, à l'issue du procès, la mère est condamnée à deux ans de prison, soit une durée inférieure à la peine minimale encourue en cas de meurtre.

Choqués par cette affaire, Yokozuka et l'association Aoi shiba no kai du département de Kanagawa décident de prendre la parole « du point de vue des assassinés 》(korosareru tachiba kara 殺される立場から), c'est-à-dire en mettant en avant le fait que, dans l'affaire concernée, l'action de la mère n'est pas seulement un acte de désespoir témoignant de sa situation de détresse, mais bel et bien un infanticide, le meurtre d'un être humain ayant une valeur en tant que tel. Ils affirment ainsi le «droit à l'existence » (seizonken 生存権) de l'enfant handicapé dont le point de vue ne peut être passé sous silence. Le premier texte signé par Yokozuka sur le sujet est une lettre ouverte, adressée notamment aux autorités judiciaires de Yokohama, dont voici un extrait :

Dans le cas de l'accusée de cette affaire, Hakamata Mihoko, on peut penser que, même si elle faisait face à de multiples difficultés matérielles néfastes pour ses deux enfants handicapés, l'idée communément répandue « que ces enfants n'avaient pas la forme qu'ils auraient dû avoir » a fait peser sur elle une charge psychique supplémentaire et l'a amenée à désespérer de leur avenir. Cependant, la vraie protection sociale ne consiste-t-elle pas à ce que chaque individu dans la société ait de la considération et du respect pour ceux qui sont différents ou plus faibles que lui ? Par exemple, la vie des enfants lourdement handicapés totalement paralysés aussi doit être respectée. Il est facile de réduire les causes de cette affaire au manque d'établissements spécialisés et à l'indigence des politiques 


\section{CIPANGO}

sociales. Si, de cette manière, on gomme le crime de l'accusée, c'està-dire si le tribunal la déclare innocente, à notre avis, cela revient dans le cas présent à promouvoir une tendance à négliger la vie des enfants/personnes handicapés et à assimiler les personnes ayant une paralysie cérébrale à des êtres qui ne devraient pas exister. Nous ne haïssons pas la mère accusée, nous ne souhaitons pas le moins du monde qu'elle reçoive une lourde peine. À l'opposé, nous pensons qu'elle est, elle aussi, une victime de la société actuelle. Néanmoins, du fait de la gravité du crime qu'elle a commis, il serait naturel qu'elle reçoive une forme de sanction.

本事件の被告袴田美保子においてもたとえ2人の障害 児を抱え幾多の生活上の困難があったにしろ、この「本 来あるべき姿ではない」という一般通念が彼女に実際以 上の精神的負担となっておおいかぶさり、子供の将来・ 自分の前途を悲観し絶望的になってしまったと思われま す。しかしながら真の社会福祉とは社会の一人一人が、 自分とは異なった姿の者、自分より弱い立場の者に対す る思いやりを持ち、その立場を尊重することではないで しょうか。たとえ寝たきりの重症児でもその生命は尊ば れなければなりません。本事件の原因を施設が足りない こと、福祉政策の貧困に帰してしまうことは簡単です。 しかしそのことによって被告の罪が消えるならば、即ち 本裁判においてもしも無罪の判決がくだされるならば、 その判例によって重症児（者）の人命軽視の風潮をます ます助長し脳性まひ者をいよいよこの世にあってはなら ない存在に追い込むことになると思われます。私達は被 告である母親を憎む気持ちはなく、ことさらに重罪に処 せというものでは毛頭ありません。それどころか彼女も また、現代社会における被害者の一人であると思われま す。しかし犯した罪の深さからいって何等かの裁きを受 けるのは当然でありましょう。16

16. Yokozuka, 2007, p. 95 (article original : « Aru shōgaisha undō no mezasu mono »). 
On trouve ici l'expression « ce n'est pas la forme qu'on devrait avoir » (honrai aru beki sugata de wa nai 本来あるべき姿ではない), qui fait écho à la «mauvaise descendance » de la loi de protection eugénique. L'association dénonce ainsi le fait que les personnes handicapées seraient perçues comme des êtres inutiles, superflus, dont l'existence n'est pas reconnue en tant que vie humaine ayant la même valeur que celle des autres personnes.

C'est à la suite de cette affaire que Yokozuka multiplie les écrits et les prises de paroles, tandis qu'Aoi shiba no kai intervient publiquement par des manifestations et des rencontres avec d'autres associations. Yokozuka développe ainsi sa pensée, sur la base de cette première réaction, fondatrice pour la suite de son action militante.

Pour lui, le «droit à l'existence » des personnes handicapées et, plus encore, le «droit d'être handicapé et de s'affirmer comme tel » doivent être opposés à une société fondée sur des bases eugénistes. Bien que Yokozuka, dans ses écrits, ne cherche jamais à donner une définition théorique de l'eugénisme, il applique ce terme à deux types de situations : la destruction physique (souvent plus supposée que réelle) des personnes handicapées et leur enfermement dans des établissements spécialisés. Pour comprendre ce qui fonde le discours d'émancipation de Yokozuka, il est donc nécessaire de revenir sur le contexte du traitement social des personnes handicapées dans les années 1960 (de quoi cherche-t-il à s'émanciper ?) et d'entrer avec précision dans sa perception et son analyse de ces deux types de situations.

\subsection{De la critique de l'eugénisme comme destruction physique...}

En ce qui concerne la destruction physique de personnes en situation de handicap, qui prend plus particulièrement la forme de l'infanticide, Yokozuka analyse les réactions suscitées par l'affaire citée ci-dessus dans un article publié en août 1970 dans le magazine associatif Ayumi (あゆみ Marche) sous le titre «Au-delà de la discrimination »(Sabetsu izen no nanika ga aru 差別以前の何かがあ る) ${ }^{17}$. Dans ce texte, il cherche à mettre en avant le fait que l'infanticide d'un enfant handicapé n'est pas seulement la conséquence de la détresse individuelle

17. Ibid., p. 80-81 (article original : Yokozuka Kōichi, « Sabetsu izen no nanika ga aru 差別以前の何かがある [Au-delà de la discrimination], Ayumi あゆみ, 10 août 1970). 
140 Cahiers d'études japonaises n² 24

d'un parent ou de la faiblesse des aides sociales, mais s'inscrit dans un contexte social plus large de discrimination envers les personnes handicapées, marqué par une hésitation sur l'humanité de celles-ci ou, tout au moins l'idée qu'elles appartiennent à un monde différent de l'existence ordinaire. Pour lui, « dans cette affaire, qu'il y ait abandon des poursuites ou acquittement, ou que l'accusée soit jugée coupable, ce sera un moment de vérité où l'on saura si la société ordinaire, à commencer par les juges, considèrent les enfants lourdement handicapés comme leurs congénères humains ( $s$ 'ils se retrouvent en eux) ou comme une espèce radicalement différente d'eux $\gg$.

Cette interrogation sur l'humanité des personnes handicapées n'est pas spécifique au Japon. Dans une perspective anthropologique plus générale, Henri-Jacques Stiker ${ }^{18}$ montre que, dans un très grand nombre de cultures, les personnes handicapées sont considérées comme appartenant à un monde différent de l'ordre ordinaire des choses, soit qu'elles soient méprisées et discriminées, c'est-à-dire traitées comme inférieures au reste de l'humanité, soit, à l'extrême inverse, qu'on leur associe des caractéristiques surhumaines et surnaturelles, les deux attitudes pouvant être entremêlées avec la plus grande ambiguïté. Pour Stiker, les personnes handicapées apparaissent aux personnes valides comme une expression de l'inquiétante étrangeté de la vie quotidienne, à la fois miroir dans lequel celles-ci se reconnaissent et vecteur d'une effrayante irrationalité, d'où des réactions où se mêlent fascination et rejet. Yokozuka souhaite que la société, et en particulier la justice, puissent s'abstraire de ces considérations pour considérer les personnes handicapées avant tout dans leur humanité, dans leur ressemblance plutôt que leur différence.

Il remet plus particulièrement en cause l'idée communément répandue que l'enfant handicapé est fondamentalement malheureux et que la mort est donc préférable à une vie dans sa situation.

Pourquoi a-t-elle (la mère meurtrière) eu ce désir de meurtre ? Pour débattre de cette affaire, on doit faire du désir de meurtre le point de départ de tout. Comme elle l'a expliqué elle-même, elle a pensé que « cette enfant n’allait pas guérir. Le bonheur, pour elle,

18.STIKER, 2005, chapitre 7. 
était donc plutôt de mourir que de vivre dans cet état ». Ce qui pose problème, c'est l'attribution d'une valeur à un être humain, selon que cette enfant guérisse ou non, qu'elle puisse travailler ou non. Dans ce système de valeurs qui exclut les personnes qui ne peuvent pas travailler, les personnes handicapées sont traitées comme des êtres qui ne devraient pas exister et subissent une oppression permanente.

なぜ彼女（子殺しの母）が殺意をもったのだろうか。
この殺意こそがこの問題を論ずる場合のすべての起点と
ならなければならない。彼女も述べているおり「この
子は治らない。こんな姿で生きているよりも死んだほう
が幸せなのだ」と思ったという。治るか治らないか、働
けるか否かによって決めようとする、この人間に対する
価值観が問題なのである。この働かざる者人に非ずとい
う価值観によって、障害者は本来あってはならない存在
とされ、日夜抑圧され続けている。19

Ainsi, Yokozuka critique le système de valeurs qui pousse les personnes valides à considérer l'existence d'une personne handicapée nécessairement comme un malheur, selon des critères qui leur sont propres (dont on verra plus bas qu'ils se fondent sur la capacité de la personne à correspondre aux besoins de la société industrielle), sans prendre en compte le ressenti de la personne elle-même. Il fait ainsi écho aux croyances entourant la naissance, dont Carolyn Stevens ${ }^{20}$ montre qu'elles sont marquées par des rituels visant à garantir la naissance d'un enfant en bonne santé, « normal », tout autre cas étant perçu comme fondamentalement malheureux. À une période où commencent à apparaître les premiers tests prénataux, la question de l'avortement sélectif se pose donc tout particulièrement. En juin 1972, une proposition de révision de la loi de protection eugénique vise à

19. Yokozuka, 2007, p. 12 (article original Yokozuka Kōichi, « CP to shite ikiru » CPとして生きる [Vivre en tant que personne ayant une paralysie cérébrale], Aoi shiba 青い芝, 1, 1972).

20. Stevens, 2013, p. 26. 
142 Cahiers d'études japonaises nº 24

supprimer la clause qui permettait d'avorter « pour des raisons économiques », ce qui était le cas pour l'immense majorité des avortements et soulève la colère des associations féministes, et dans le même temps d'ajouter une clause rendant légal l'avortement explicitement lié à la détection d'une anomalie du fotus (devenue possible grâce au développement des diagnostics prénataux). Pour Yokozuka, il s'agit là d'une mesure très dangereuse susceptible d'aggraver encore la discrimination subie par les personnes handicapées :

Selon cette loi, du moment où l'on identifie l'enfant comme « handicapé », en remontant jusqu'à l'intérieur de l'utérus de la mère, il devient possible et parfaitement légitime de le tuer sous couvert de légalité. Ce qu'on appelle dans cette loi une mauvaise descendance, du point de vue de qui est-elle donc mauvaise ? Dans la société productiviste, il s'agit de tuer les personnes handicapées ayant une faible capacité de travail qui sont des parasites pour la société et des êtres (mauvais) qui ne devraient pas exister. [...] La proposition de révision de la loi de protection eugénique qui justifie légalement cette logique meurtrière diffère-t-elle fondamentalement de la politique de l'Allemagne nazie qui, en plus du génocide des Juifs, a fièrement assassiné des centaines de milliers de personnes ayant un handicap physique ou mental, au nom du renforcement du peuple germain (afin d'éliminer les progénitures inférieures) ?

この改正案によると「障害児」と分かったとたん、し かも母親の胎内にまでさかのぼった状態で天下晴れて「 合法」の名のもとに抹殺できるわけです。この法律でい うところの不良な子孫とは一体誰にとっての不良なので しょうか。生産第一主義の社会においては、生産力に之 しい障害者は社会の厄介者・あってはならない存在性（

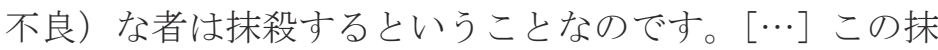
殺する論理を法律によって正当化する優生保護法改正案 は、かつてナチスドイツがユダヤ人大量虐殺とともに、 誇り高きゲルマン民族の強化という大義名分のもとに（ 


\section{劣悪な子孫をなくすため) 数十万の身体障害者、精薄者 を殺したことと基本的にどこが違うのでしょうか。21}

L'amalgame entre la politique eugénique japonaise d'après-guerre et la politique d'extermination nazie a été déconstruite par les historiens des sciences à partir des années $1990^{22}$. Néanmoins, s'il apparaît aujourd'hui comme un raccourci contestable au point de vue des faits, il exprime, chez Yokozuka, une profonde peur du renforcement des discriminations. Dans la position d'infériorité et d'impossibilité à se faire entendre et à se défendre qui est alors celle des personnes handicapées, l'argument s'inscrit dans la logique d'une pensée fondée sur la conscience d'être considéré comme « des êtres qui ne devraient pas exister ». Pour Yokozuka, la suspicion d'une visée exterminatrice est la conséquence logique d'une argumentation née de la dénonciation d'un meurtre.

\section{2. ... À la critique des pratiques ségrégatives}

Au-delà de l'extermination physique des personnes handicapées qui demeure le plus souvent de l'ordre de l'hypothèse, en dehors des affaires d'infanticide qui ont donné naissance au mouvement, Yokozuka dénonce les conditions de vie des personnes handicapées dans les établissements spécialisés. Rappelons que la période est à la construction de très grands établissements, dits koronīコロニー, qui accueillent entre 200 et 800 résidents atteints de handicaps lourds, physiques et mentaux. L'initiative de la construction de ces établissements est lancée à partir de 1966. Ceux-ci sont conçus comme des foyers où les personnes lourdement handicapées peuvent vivre ensemble, s'entraider et recevoir des soins rééducatifs (quoiqu'il s'agisse en principe d'un environnement peu médicalisé), ainsi qu'une formation professionnelle. L'un des objectifs de leur création est la volonté d'offrir aux personnes ayant un handicap lourd ou plusieurs handicaps des conditions de vie décentes et des soins adaptés, tout permettant aux familles de ne plus porter

21. Yokozuka Kōichi, 2007, p. 129-131 (article original : Yokozuka Kōichi, « Yūsei hogo-hō to watashi » 優生保護法と私 [La loi relative à la protection eugénique et moi], Ayumi あゆみ, 16, septembre 1972).

22.Voir à ce sujet Matsubara \& ICHINOKAwA, 2000. 
seules la responsabilité de la prise en charge de leurs membres handicapés. Il s'agit donc notamment d'assurer la continuité des écoles spécialisées qui se développent dans tout le pays (bien que l'éducation ne soit pas encore obligatoire pour tous les enfants en situation de handicap) et d'assurer une prise en charge spécialisée des adultes handicapés. En 1971, on compte 19 établissements de ce type qui accueillent 8909 résidents, sur une population totale identifiée de 11359 personnes vivant avec un handicap physique et mental lourd, soit un taux de vie en institution spécialisée de 78,4\% pour ces personnes ${ }^{23}$.

Pour Yokozuka, il s'agit là d'une autre facette, moins explicite, de l'eugénisme dans la société : les établissements spécialisés auraient avant tout une visée ségrégative. Ils seraient construits dans le but d'exclure les personnes handicapées du reste de la société, sans que cela s'inscrive dans un souci réel de leurs conditions d'existence. C'est pourquoi il les compare fréquemment à des prisons, voire à des camps de concentration. Notons cependant que cela ne correspond pas à l'objectif explicite de la politique de création des établissements : par opposition aux établissements accueillant les lépreux qui s'inscrivent ouvertement dans une logique de mise en quarantaine de cette population afin d'éviter de supposés risques de contamination, les établissements destinés aux personnes handicapées sont présentés comme visant à leur réadaptation (notamment par la formation professionnelle) et à leur 《retour à la société 》 (shakai fukki 社会復帰), même si, en pratique, ce « retour » se produit rarement. Si logique d'exclusion il y a, elle est donc implicite et non directement inscrite dans la loi, comme elle peut l'être pour d'autres populations.

Yokozuka donne d'un établissement spécialisé la description suivante :

Au centre de Fuchū, cinquante personnes sont enfermées dans une pièce, la porte d'entrée est habituellement fermée à clé et les membres du personnel retirent la clé à chacune de leurs entrées et sorties. Derrière la porte d'entrée, il y a le bureau du personnel, et de là on peut surveiller la pièce où se trouvent les cinquante personnes. Les personnes venues de l'extérieur ne peuvent pas passer cette porte. Les personnes handicapées qui sont à l'intérieur

23.ENDŌ, 2014. 
portent un pyjama uniforme (le chauffage est allumé et il ne fait pas froid) et ne possèdent presque pas d'objets personnels. Pour sortir ou dormir à l'extérieur, il faut que leurs parents remplissent un formulaire de demande d'autorisation deux semaines à l'avance et personne d'autre que les parents ne peut obtenir la permission. $\grave{A}$ part cela, comme les cinquante personnes sont entassées ensemble, elles n'ont ni le temps ni l'espace d'être seul et de penser, leur liberté individuelle est complètement niée.

府中センターでは一部屋で五十人ずつ収容され、入口 には常に鍵がかけられ、職員の出入にもいちいち鍵をか けはずしする。入り口の内側に職員の詰所があり、そこ から五十人入りの部屋が一望できるようになっている。 外来者はその入り口までしか行くことができない。中に いる障害者はお揃いのパジャマ一枚（暖房付きの為寒 くはないが）で、私物は殆ど持たされない。外泊、外出 は親が二週間前に申し入れを行い許可を得なければなら ず、保護者以外では許可されない。そのほか、五十人も 一緒に詰め込まれているため、ひとりになってものを考 える時間も空間もなく、個人的自由など全く無視されて いるとのことである。 24

Les conditions de vie, ainsi décrites ${ }^{25}$, rappellent en effet fortement l'univers carcéral, un univers qui dépossède l'individu de lui-même et le prive de toute liberté. C'est pourquoi, à la place du mot 《établissements » (shisetsu 施設), Yokozuka emploie très fréquemment le terme plus ambigu de shōgaisha shīyō shisetsu 障害者収容施設, que l'on pourrait traduire aussi bien par « foyers de vie » que par «centres de détention de personnes handicapées ». D'ailleurs, il note dans l'un de ses textes que, fréquemment, lorsqu'il se promène seul dans la rue, il sent peser sur lui les regards et entend des questions telles que « à quel établissement appartient-il ? », quand ce n'est pas « de quel établissement s'est-il

24. Yoкоzuka, 2007, p. 78 (article original : « Sabetsu izen no nanika ga aru »).

25. Ibid., p. 78 (article original : « Sabetsu izen no nanika ga aru »). 
enfuii ${ }^{26}$ ? ». Il fait ainsi apparaître très nettement l'équation : établissement spécialisé = privation de liberté et d'individualité avec l'assentiment du reste de la société. D'où l'analogie avec l'univers concentrationnaire, lorsque Yokozuka affirme que les grands établissements, version moderne et collective des chambres de confinement (zashikirō 座敷牢) de la période d'Edo, participent de la même logique que le camp d'Auschwitz pour l'Allemagne hitlérienne : celle de la mobilisation nationale au détriment des plus faibles ${ }^{27}$.

Si Yokozuka n'exprime pas explicitement l'idée d'une visée génocidaire de l'État japonais (ce qui irait contre la réalité des faits), il dénonce néanmoins une volonté d'oppression qui, pour lui, justifie la comparaison avec l'Allemagne nazie. Les établissements ont, selon lui, vocation à exclure de la société tous ceux qui ne sont pas utiles à cette nouvelle forme de mobilisation nationale qu'est le développement économique de la période de Haute Croissance. Il s'agit donc d'une visée ségrégative, permettant au reste de la société de se présenter sous son meilleur jour en dissimulant l'existence des personnes indésirables. Celles-ci sont alors livrées à elles-mêmes, dans des conditions de vie rudimentaires, ne recevant en soutien que le minimum vital. Leur intégrité physique peut même être directement atteinte, comme dans le cas des opérations de stérilisation eugénique ${ }^{28}$ qui y sont pratiquées ${ }^{29}$. D'où cette conclusion lapidaire :

On dit aussi que «Plutôt que de punir la mère qui a tué son enfant handicapé, il faut d'abord construire des établissements ». Mais il est impossible que les établissements qui sont construits sur la base de telles pensées soient liés au bonheur des personnes handicapées. En effet, ce sont des choses qui ont été créées en dissimulant le fait qu'on vole la vie d'enfants handicapés et en mettant en péril le droit à l'existence des personnes handicapées. Ici se cachent, sous le nom de protection sociale, l'égoïsme et les pensées discriminatoires des parents et de la société.

26. Ibid., p. 103 (article original : « Aru shōgaisha undō no mezasu mono »).

27. Ibid., p. 79 (article original : « Sabetsu izen no nanika ga aru »).

28. Ibid., p. 104 (article original : « Aru shōgaisha undō no mezasu mono »).

29. Sur le contexte social et la pratique de ces opérations, voir TOSHIMITsu, 2016. 
「重症児を殺した母親を罰するよりもまず収容施設を つくることだ」とも言われます。しかしこのような発想 から作られる施設が、障害者の幸せにつながる筈はあり ません。なぜならそれは重症児の生命を奪ったことを曖 昧にし、障害者の生存権をも危うくする思想から作られ るものだからです。ここにこそ福祉に名を借りた親たち や社会のエゴイズムと差別意識が潜んでいるのです。 30

Yokozuka dénonce donc, avec des références insistantes au totalitarisme nazi, un eugénisme menaçant plutôt que protecteur. Mêlant dans un même mouvement de pensée ségrégation en établissement spécialisé et extermination physique, il dénonce, plus que la pratique réelle de l'eugénisme, un système social qui prive les personnes handicapées de leur liberté et de leurs droits individuels. Pour appuyer sa critique, il cherche à analyser les causes de cette situation et la nature de l'oppression dite « eugéniste ».

\section{Au cœur de la société : «l'eugénisme intériorisé »}

Pour Yokozuka, l'eugénisme présent au cœur du fonctionnement de la société prend plusieurs formes : il est non seulement explicite, c'est-à-dire découlant de lois à la visée eugénique affirmée (au premier rang desquelles la loi de protection eugénique de 1948), mais aussi insidieux, lié à l'intériorisation par tous les membres de la société (y compris les personnes handicapées elles-mêmes et leurs familles) de normes de rationalité et d'efficacité directement liées à l'industrialisation. C'est cette double nature de l'eugénisme qu'il analyse dans ses écrits.

Les effets directs de la loi de 1948 sont connus : au cours du demi-siècle d'application de la loi, environ 16500 opérations de stérilisation forcée de personnes susceptibles d'avoir une descendance indésirable ont été officiellement comptabilisées $^{31}$ et des groupes de population ont été mis en quarantaine (notamment les lépreux) sans possibilité de retour à la vie ordinaire. Cependant, la

30. Yokozuka, 2007, p. 101 (article original : « Aru shōgaisha undō no mezasu mono $\gg)$.

31. Matsubara, 2000, p. 171. 


\section{CIPANGO}

148 Cahiers d'études japonaises n² 24

dénonciation de l'eugénisme explicite, mis en place par l'État par l'intermédiaire de lois, occupe paradoxalement peu de place dans les écrits de Yokozuka.

Des passages sur les stérilisations pratiquées en établissement spécialisé ou sur l'avortement sélectif, comme ceux cités ci-dessus, montrent clairement son opposition au système mis en place par la loi de protection eugénique et aux tentatives de renforcement de celui-ci. Les actions militantes menées par Aoi shiba no kai visent également clairement à lutter contre l'eugénisme d'État, qu'il s'agisse de manifestations, de négociations avec les autorités ou de campagnes médiatiques. Mais, dans ses écrits, Yokozuka veut surtout affirmer que, bien que la dénonciation des lois et du système politique en vigueur soit nécessaire, elle n'est pas suffisante et ne peut faire l'économie d'une critique sociale plus globale, au-delà de la remise en question de la seule loi de protection eugénique. C'est pourquoi beaucoup de ses textes visent à mettre en lumière un eugénisme intériorisé présent au sein de la société, y compris chez les personnes handicapées elles-mêmes, eugénisme fondé sur les normes de l'industrie.

En effet, comme le dénoncera également plus tard Michael Oliver ${ }^{32}$, l'un des fondateurs des disability studies anglo-saxonnes, l'avènement de la société industrielle a contribué à une sélection nette entre des personnes « utiles » (ayant la capacité physique et psychique de travailler) et les personnes « inutiles » (ne pouvant servir de main d'œuvre dans l'industrie). Yokozuka voit donc dans l'industrie et le productivisme la principale cause de la marginalisation des personnes handicapées et de la négation de leur « utilité » sociale.

Dans les sociétés qui, comme le Japon, visent à un fort développement économique, on exige le plus rapide, le moins cher, le plus beau, et il est certain que les personnes handicapées, dont l'efficacité est faible et l'apparence mauvaise, sont au plus bas de la hiérarchie.

$$
\begin{array}{r}
\text { 日本のように高度経済成長を目的とする社会において } \\
\text { は、いかに早く、いかに安く、いかにみかけがよくとい }
\end{array}
$$

32. Oliver, 1990. 
うことが要求され、能率の悪い、みかけの悪い障害者が 下の下であることは確かです。33

Ainsi, pour Yokozuka, l'industrialisation a fortement contribué à placer les personnes handicapées en position d'infériorité dans la société. Il évoque un « autrefois » idéalisé où les personnes âgées ou handicapées prenaient en charge des rôles tels que gardiens du feu ou intermédiaires avec le monde des esprits ${ }^{34}$, ce qui leur conférait une utilité sociale, indépendamment de leurs capacités physiques. L'industrialisation, en imposant des normes d'efficacité, a contribué à la rationalisation de la société et à la standardisation des corps et des esprits. Il est alors impossible pour les personnes handicapées de trouver une place dans ce système et leur seule défense possible est alors de s'affirmer en tant « qu'êtres irrationnels », à l'encontre de ces normes.

Afin d'augmenter la production et les profits, la société rationnelle moderne accélère le rythme de sa tendance à la rationalisation, à la division fonctionnelle, à la standardisation. Cependant, il est naturel qu'il y ait une opposition à cela, car l'homme est depuis toujours irrationnel. [...] En tout cas, les personnes handicapées sont l'archétype de l'être irrationnel [...] Si nous cessons de vénérer inutilement « les valides » et de les envier, tandis que le système social « valide » continue de se ruer vers la rationalisation sans refléter l'humanité et de nous exclure, nous personnes handicapées qui ne correspondons pas aux standards, nous devrons continuer notre mouvement jusqu'au bout en nous appuyant sur notre conscience d'être des êtres irrationnels.

近代合理社会は生産性を高め、利潤を上げるためにま すます合理化、機能化、画一化への方向へ加速度を強め

33. Yoкоzuka, 2007, p. 53-55 (article original : Yokozuka Kōichi, « Shōgaisha to rōdō » 障害者と労働 [Les personnes handicapées et le travail], Manuscrit d'une conférence donnée à l'université de Tōkyō, mars 1972).

34. Ibid., p. 55 (article original : « Shōgaisha to rōdō »). 


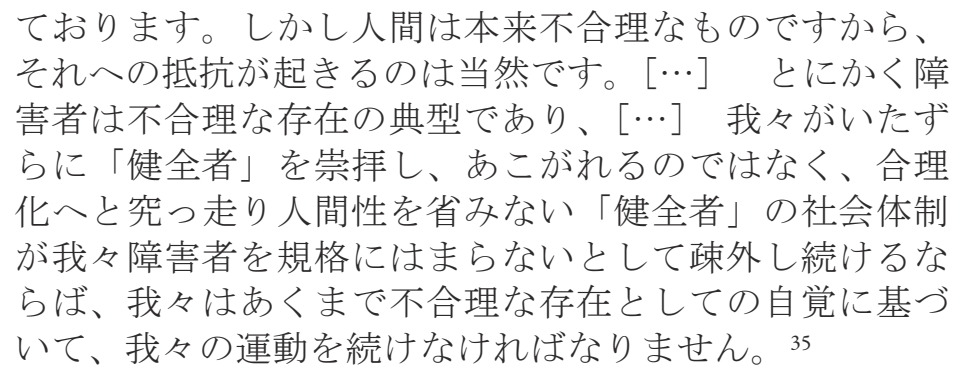

Ainsi, cette conscience d'appartenir à une partie de l'espèce humaine que la société voudrait éliminer amène Yokozuka à envisager l'affirmation de soi, c'est-àdire l'affirmation identitaire des personnes handicapées en tant que telles, comme seule réponse possible à la discrimination.

\section{Un « grand cri »: l'affirmation identitaire comme réponse à l'eugénisme}

La nécessité de l'affirmation identitaire prônée par Yokozuka est profondément ancrée dans son expérience du bouddhisme et de la vie en communauté religieuse ${ }^{36}$, évoquée en introduction. La communauté en question portait le nom de « village Maharaba » : Mahārāva est un mot sanscrit qui signifie « grand cri ». Ce grand cri s'inscrit dans la pensée du prêtre Osaragi Akira, inspirée des écrits de Shinran ${ }^{37}$ : Osaragi reconnaît que l'être humain est, par nature, profondément marqué par le mal, c'est-à-dire qu'il lui est impossible de vivre sans faire de mal à autrui. La première étape vers l'illumination consiste donc à accepter cet aspect de la nature humaine, à prendre conscience de cette part de soi, et à adresser au bouddha Amida une demande d'aide, un cri, en scandant le nenbutsu ${ }^{38}$. Pour Yokozuka, il est donc nécessaire que les personnes handicapées ne se définissent

35. Ibid., p. 84-85 (article original : Yokozuka Kōichi, « Fugōri na sonzai to shite » 不合理な存在として [En tant qu'êtres irrationels], Aoi shiba 青い芝, 93, mars 1974).

36. YORITAKA, 2012, p. 52.

37. Shinran (親鸞) (1173-1262) est le fondateur de l'école bouddhique Jōdo shinshū.

38. Ibid., p. 113 (article original : « Aru shōgaisha undō no mezasu mono »). 
pas exclusivement comme victimes, comme oppressées, mais qu'elles prennent conscience qu'elles sont aussi des êtres humains à part entière, donc susceptibles de faire du mal. Cette interrogation sur soi est le premier pas vers l'affirmation de soi (jiko shuchō 自己主張) à laquelle appelle Yokozuka, affirmation qui est la seule manière de s'extraire de la menace qui pèse sur leur existence ${ }^{39}$.

Yokozuka montre qu'avant même d'affirmer leur existence face à la société ordinaire, les personnes handicapées doivent prendre conscience d'elles-mêmes et rejeter les normes des valides qu'elles ont intériorisées. Il s'agit en premier lieu d'un travail sur soi, un travail de réflexion et de prise de conscience. C'est pourquoi Yokozuka insiste sur l'importance de la subjectivité : avant de chercher à participer aux activités de la société ordinaire, il est nécessaire de développer une personnalité, une subjectivité individuelle ${ }^{40}$, de se reconnaître soi-même comme individu ayant le droit d'exister au même titre que les autres. Il s'agit donc de se défaire des illusions à propos des valides et de leur prétendue supériorité ${ }^{41}$ et de surmonter non pas le handicap (ce qui supposerait l'existence d'une norme universelle vers laquelle il faudrait chercher à tendre ${ }^{42}$ ) mais le sentiment de perte et d'infériorité qui lui est associé dans la société moderne. L'affirmation individuelle est donc le point de départ du mouvement. C'est un « grand cri » qui prend racine dans la pratique de la récitation du nenbutsu telle que la conçoit Osaragi Akira.

Ce « grand cri » doit alors être entendu par la société et, pour cela, il est nécessaire que les personnes handicapées sortent dans la rue, se montrent, se fassent entendre.

39. Ibid., p. 97 (article original : « Aru shōgaisha undō no mezasu mono »).

40. Ibid., p. 89 (article original : Yokozuka Kōichi, « Nōsei mahisha no shakai sanka nit suite »脳性まひ者の社会参加について $[\AA$ A propos de la participation sociale des personnes ayant une paralysie cérébrale], Aoi shiba, 77, avril 1970).

41. Ibid., p. 63-66 (article original : Yokozuka Kōichi, « Nōsei mahi to shite ikiru » 脳 性マヒとして生きる [Vivre en tant que paralysé cérébral], Conférence à l'Université municipale de Kawasaki, avril 1972).

42. Ibid., p. 75 (article original : YokozuKa Kōichi, « N jo e no henshin » N女への返 信 [Réponse à Mme N.], Ayumi, 17, janvier 1973). 
152 Cahiers d'études japonaises nº 24

À ce propos les trains, les bus, les passerelles pour les piétons, les escaliers des gares et encore d'autres constructions architecturales, les villes entières ignorent notre existence et nient le fait que nous y vivons. Alors j'ai pensé qu'il fallait que notre mouvement commence par le fait de sortir dans les villes. C'est cela nous exposer tels que nous sommes, c'est cela une affirmation de soi forte.

そういえば電車、バス、歩道橋、駅の階段やいろいろ の建築物など町そのものが私達の存在を無視し、そこで 私達が生きていくことを拒否しているのです。そこで私 達の運動は街に出ることから始めなければならないと考 えたのです。それは私達のありのままをさらけだすこと であり、強烈自己主張であります。43

Cette affirmation de soi passe en particulier par l'expression artistique ${ }^{44}$, mais aussi, à l'heure des stérilisations forcées, par le fait d'avoir des enfants ${ }^{45}$, comme Yokozuka lui-même et ses camarades du village Maharaba, malgré les difficultés matérielles certaines liées à la parentalité (la revendication de Yokozuka n'est donc plus seulement celle du droit à la naissance, mais du droit à la reproduction). Cette affirmation de soi est rendue particulièrement difficile par le fait que la société moderne établit un lien fort entre valeur individuelle et travail ${ }^{46}$, mais il est nécessaire de lutter contre la standardisation et contre une société qui voudrait « faire rentrer tous les pieds dans une chaussure préfabriquée et couper ceux qui n'y rentrent pas pour les y faire entrer de force ${ }^{47} \gg$.

C'est cette logique d'affirmation qui pousse Aoi shiba no kai à multiplier les actions publiques et médiatiques dans la première moitié des années $1970^{48}$.

43. Ibid., p. 63 (article original : « Nōsei mahi to shite ikiru »).

44. Ibid., p. 66 (article original : « Nōsei mahi to shite ikiru »).

45. Ibid., p. 74-75 (article original : « N jo e no henshin »).

46. Ibid., p. 90 (article original : « Nōsei mahisha no shakai sanka ni tsuite »).

47. Ibid., p. 91 (article original : « Nōsei mahisha no shakai sanka ni tsuite »).

48. Ibid., p. 82 (article original : « Fugōri na sonzai to shite »). 
Leur action la plus emblématique demeure la rédaction, en 1970, du manifeste de l'association ${ }^{49}$ :

- Nous nous reconnaissons en tant que personnes ayant une paralysie cérébrale. Nous avons conscience d'être, dans la société actuelle, des « personnes qui ne devraient pas exister », nous croyons qu'il faut faire de cela le point de départ de tout notre mouvement, et nous agissons en conséquence.

- Nous nous affirmons avec force. Quand nous nous reconnaissons en tant que personnes ayant une paralysie cérébrale, ce qui en résulte pour nous est la volonté de nous protéger. Nous croyons qu'une affirmation de soi forte est la seule voie possible pour nous, et nous agissons en conséquence.

- Nous refusons l'amour et la justice. Nous dénonçons vivement l'égoïsme qui prend le nom de l'amour et de la justice, nous croyons que seule la compréhension mutuelle accompagnée d'un regard humain fondé sur ce refus constitue une véritable protection sociale, et nous agissons en conséquence.

- Nous ne choisissons pas la voie de la résolution des problèmes. Nous avons appris par nous-mêmes que chercher à résoudre les problèmes de manière simpliste peut mener à des compromis dangereux. Nous croyons qu'exposer les problèmes un à un est la seule voie possible pour notre mouvement, et nous agissons en conséquence.

われらは自らがCP者であることを自覚する。我々は現 代社会にあって「本来あってはならない存在」とされつ つある自らの位置を認識し、そこに一切の運動の原点を かけなければならないと信じ、且つ行動する。

49. Manifeste rédigé non par Yokozuka lui-même, mais par l'un des autres leaders de l'association, Yokota Hiroshi 横田弘, qui a lui aussi participé à l'expérience du village Maharaba et milite aux côtés de Yokozuka dès les débuts du mouvement. 
154 Cahiers d'études japonaises nº 24

われらは強烈な自己主張を行う。われらがCP者である ことを自覚したとき、そこに起こるのは自らを守ろうと する意志である。われらは強烈な自己主張こそこれを成 し得る唯一の路であると信じ、且つ行動する。

われらは愛と正義を否定する。われらは愛と正義のも つエゴイズムを鋭く告発し、それを否定することによっ て生じる人間凝視に伴う相互理解こそ真の福祉であると 信じ、且つ行動する。

われらは問題解決の路を選ばない。われらは安易に問 題の解決を図ろうとすることがいかに危険な妥協への出 発であるか、身をもって知ってきた。われらは、次々と 問題提起を行うことのみわれらの行いうる運動であると 信じ、且つ行動する。50

Ce manifeste reprend les principaux arguments exposés par Yokozuka dans ses écrits. On peut noter que le célèbre troisième point, le « refus de l'amour et de la justice », contient une critique de nature religieuse. En effet, jusqu'alors, le système de protection sociale de personnes handicapées s'est construit dans une logique « d'aide aux plus faibles », qu'il s'agisse de compassion bouddhique (dans le prolongement de la longue tradition qui a marqué l'histoire des personnes handicapées au Japon) ou de charité chrétienne (les initiatives d'inspiration chrétiennes, notamment en matière de création d'établissements privés, ayant été très nombreuses au Japon depuis l'ère Meiji). Or, pour Yokozuka, inspiré par la pensée religieuse d'Osaragi Akira, cette logique repose sur une illusion : celle que les valides représenteraient le Bien et les personnes handicapées auraient besoin d'être secourues car elles seraient touchées par le Mal (du fait qu'elles suscitent le rejet et sont considérées comme des créatures qui ne devraient pas exister). Pour Yokozuka, cette représentation binaire masque le fait que chaque être humain porte en lui une part criminelle (zaiakusei 罪悪性). Or, c'est seulement en reconnaissant ses propres crimes, sa propre méchanceté, et en faisant de cet aveu le point de départ de sa volonté de changer le monde, que l'individu peut venir

50. Cité dans Yoritaka, 2012, p. 70. 
en aide à autrui sur la base d'une véritable solidarités1. À cet égard, Yokozuka ne se contente pas de renverser la logique en accusant les valides d'être mauvais, il montre que les personnes handicapées aussi doivent faire cet effort d'interrogation de soi et admettre leur part criminelle (notamment dans leurs interactions avec des personnes ayant un handicap plus lourd que le leur), afin que se mette en place un système de protection et d'entraide fondé sur une logique égalitaire.

Le manifeste s'appuie sur une conscience forte d'être un groupe minoritaire menacé par la société et répond à cette menace par l'affirmation de soi, définie comme seule réponse possible à l'eugénisme, explicite ou latent. Or cette voie de l'affirmation de soi choisie par le mouvement exclut toute forme de compromis. Il s'agit donc de résoudre les problèmes, non pas par des négociations, mais par une exposition et une dénonciation systématique qui peut aboutir à des confrontations.

En effet, la critique de Yokozuka soulève des réactions variées, à la mesure de sa radicalité. Trois groupes sociaux qui auraient pu apparaître comme des alliés naturels du mouvement sont dénoncés comme « oppresseurs ». Premièrement, les parents et leurs associations sont accusés non seulement de confisquer la parole des personnes handicapées, mais aussi de faire peser sur les enfants handicapés, dès leur naissance, un sentiment d'infériorité par rapport à leurs frères et sœurs valides ${ }^{52}$. Cette accusation est douloureusement vécue par les familles militantes, prises entre la discrimination dont elles font l'objet dans la société du fait du handicap de leurs enfants, et le ressentiment de ces derniers ${ }^{53}$. Deuxièmement, cette critique envers les parents est en réalité dirigée plus spécifiquement contre les mères, comme en témoigne le slogan Haha yo! Korosu na (母よ! 殺すな «Maman! Ne me tue pas »), ce qui génère des tensions avec les associations féministes, en particulier lors des débats autour de la question de l'avortement à l'occasion de la proposition de révision de la loi de protection eugénique de $1972^{54}$. Troisièmement, le corps médical et les professionnels des établissements spécialisés sont accusés de se faire complice d'un système qui nie l'humanité et l'individualité des personnes handicapées. Cependant, cette période cö̈ncide avec

51. YAMAZAKI, 2010, p. 10.

52. Yokozuka, 2007, p. 73 (article original : « N jo e no henshin »).

53. HORI, 2014.

54. KaTō, 2009. 
156 Cahiers d'études japonaises nº 24

un mouvement de renouveau dans les facultés de médecine (où naît le mouvement étudiant de la fin des années 1960) : la jeune génération de psychologues et de professionnels de santé est alors à la recherche de nouvelles méthodes de travail visant à développer l'autonomie des patients ${ }^{55}$. Ainsi, paradoxalement, si les professionnels travaillant en établissement spécialisés sont relativement prompts à reconnaitre les effets potentiellement néfastes de leur expertise et à se lancer dans un travail conjoint, valorisant l'individualité et l'humanité de leurs patients, d'autres groupes «d'oppresseurs » acceptent moins bien la critique, sont déconcertés, voire choqués par sa virulence. Bien que le combat d'Aoi shiba no kai s'inscrive dans un contexte d'émergence générale de mouvements de contestation sociale, la convergence des luttes se révèle initialement difficile et il faudra attendre une décennie avant que des terrains d'entente puissent être trouvés entre associations de personnes handicapées et associations féministes ou associations de parents. Cela reflète à la fois la prégnance dans la société des normes « d'eugénisme intériorisé » que dénonce Yokozuka et la difficulté de construire une critique unifiée de l'eugénisme faisant converger les différents mouvements de contestation, à l'image de l'ambiguïté du concept d'eugénisme lui-même et des multiples utilisations qui en sont faites.

Enfin, cet « oppresseur » moins incarné qu'est l'État intègre lui aussi progressivement, quoique lentement, les critiques de Yokozuka au nom des personnes handicapées. Ainsi, à partir du milieu des années 1970, des initiatives municipales commencent à voir le jour pour favoriser l'accessibilité des transports et des infrastructures publiques. À partir des années 1980, des politiques d'intégration commenceront à voir le jour, en réponse à la demande de désinstitutionalisation du handicap, pour permettre aux personnes handicapées de vivre hors des établissements spécialisés, dans la société ordinaire. Mais il faudra attendre 1996 pour assister à une révision complète de la loi de protection eugénique actant la fin des stérilisations forcées.

55. HORI, 2014, chapitre 4. 


\section{Conclusion}

Ce travail visait à analyser l'eugénisme au Japon au prisme de la critique qu'en font les personnes handicapées (c'est-à-dire, en leur nom, Yokozuka Kōichi) au début des années 1970, à cette période charnière où la parole de celles-ci émerge pour la première fois sur la scène médiatique et politique, afin de mettre en lumière comment la critique de l'eugénisme a constitué un argument-clé de la lutte pour l'émancipation des personnes handicapées.

On a montré que cette critique s'appuie sur une définition de l'eugénisme en deux dimensions: une critique de la menace d'extermination physique des personnes handicapées, quoique celle-ci, dans les faits, ne soit en rien comparable à ce qui s'est produit dans l'Allemagne nazie (décrit avec minutie dans l'étude de Götz Aly ${ }^{56}$ ), matérialisée par des cas d'infanticide et l'émergence des diagnostics prénataux ouvrant la voie à l'avortement sélectif; et une dénonciation de la ségrégation des personnes handicapées dans des établissements spécialisés aux conditions de vie proches de l'univers carcéral (politique qui n'est pas « eugéniste » à proprement parler). Elle analyse, à l'origine de ces deux phénomènes, d'une part un eugénisme d'État, promu en particulier par la loi de protection eugénique de 1948 (qui implique notamment des stérilisations forcées) et, d'autre part, un eugénisme plus implicite, omniprésent dans les relations sociales, issu de l'intériorisation des normes d'efficacité et de rationalité liées aux besoins de l'industrie. Pour Yokozuka, la seule réponse possible à cet eugénisme est l'affirmation de soi, l'affirmation identitaire des personnes handicapées en tant que telles, la mise en avant de leur existence niée dans le fonctionnement de la société ordinaire et la confrontation avec d'autres groupes sociaux dénoncés comme des « oppresseurs ». Ainsi, son argumentaire passe d'une revendication d'un droit à l'existence (contre une société perçue comme souhaitant la disparition des personnes handicapées) à un droit à la reconnaissance (du handicap en tant que spécificité individuelle, revendiquée en tant que telle et non plus vécue dans la honte du stigmate). De la critique de l'eugénisme naît donc un discours d'émancipation qui dépasse le contexte de la politique eugénique en vigueur à cette période.

56. Aly, 2014. 
158 Cahiers d'études japonaises nº 24

Ce discours, par sa radicalité, soulève des réactions variées et parfois virulentes. $\mathrm{Si}$ les professionnels des établissements spécialisés se prêtent rapidement à l'autocritique, les relations avec les associations de parents demeurent tendues pendant près d'une décennie, tandis qu'un conflit ouvert éclate avec les associations féministes, alors même que l'opposition des deux groupes au projet de révision de la loi de protection eugénique aurait pu être un terrain propice à une fédération des luttes.

Cependant, cette critique, malgré l'accueil mitigé qu'elle reçoit initialement, joue un rôle central dans l'histoire de l'eugénisme japonais. En effet, elle contribue fortement au passage d'une conception politique « préventive » de l'eugénisme comme instrument de l'amélioration de la population et de ses conditions de vie (conception dont témoigne la formulation de la loi de 1948 et la présence de l'expression « protection eugénique » dans son titre) à une vision plus réprobatrice, ancrée dans des références à l'Allemagne nazie et marquée par l'idée de l'eugénisme comme menace, un passage qui sera sanctionné en 1996 par la suppression des termes relatifs à l'eugénisme dans la loi totalement révisée qui deviendra la loi de protection du corps des mères (Bōtai hogo-hō 母体保護法). Les effets de cette dénonciation sont visibles encore aujourd'hui, dans l'amalgame qui demeure courant entre « eugénisme » et oppression des personnes handicapées.

De plus, au-delà de son influence sur les lois et la politique japonaises, ce discours parvient à toucher ses principaux destinataires qui ne sont finalement ni l'État japonais, ni la société valide, mais les personnes handicapées elles-mêmes, en leur faisant pour la première fois prendre conscience que leur voix mérite d'être entendue. Il apparaît comme une révélation pour les jeunes en situation de handicap atteignant l'âge adulte à cette période, à qui il inspire la confiance en soi, la volonté d'accepter son handicap comme une identité plutôt que de le vivre dans la honte. Ainsi, dans sa virulence et dans ses excès mêmes, il constitue, plus encore qu'un témoignage sur la politique eugénique d'après-guerre, un discours politique inédit porteur d'émancipation. 


\section{Bibliographie}

AsAKA Yūho 安積遊歩, 1993, Iyashi no sekushī torippu 癒しのセクシートリ ップ (Sexy trip thérapeutique), Tarōjirōsha 太郎次郎社, Tōkyō, 232 p.

Aly Götz, 2014, Les Anormaux, Flammarion, Paris, 310 p.

ENDŌ Hiroshi 遠藤浩, 2014, Kokuritsu koronī kaisetsu ni itaru michinori 国立 コロニー開設に至る道のり (Vers l'ouverture des grands établissements nationaux pour personnes handicapées), Bulletin commémoratif des 10 ans de l'établissement Nozomi no Sono, 36 p. (URL : http://www.nozomi.go.jp/ publication/PDF/kiyou_10year_01.pdf,_consulté le 10/05/2016).

Hara Kazuo, 2009, Camera Obtrusa: The Action Documentaries of Hara Kazuo, Kaya, 400 p.

Hori Tomohisa 堀智久, 2014, Shōgaigaku no aidentiti - Nihon ni okeru shōgaisha undō no rekishi kara 障害学のアイデンティティ - 日本におけ る障害者運動の歴史から (L'identité des études sur le handicap - à partir de l'histoire des mouvements de personnes handicapées au Japon), Seikatsu shoin 生活書院, Tōkyō, $224 \mathrm{p}$.

Kato Masae, 2009, Women's Rights?: The Politics of Eugenic Abortion in Modern Japan, Amsterdam University Press, Amsterdam, 245 p.

Matsubara Yōko 松原洋子, 2000, Nihon - Sengo no yūsei hogo-hō to iu na no danshu-hō 日本一戦後の優生保護法という名の断種法（Japon - après-guerre, une loi de stérilisation sous le nom de loi de protection eugénique), in Yonemoto Shōhei 米本昌平, Nudeshima Jirō 橳島次 郎, MATsubara Yōko 松原洋子, Ichinokawa Yasutaka 市野川容孝 (dir.), Yüseigaku to ningen shakai 優生学と人間社会 (Eugénisme et sociétés humaines), Kōdansha gendai shinsho講談社現代新書, Tōkyō, p. 170-236. 


\section{CIPANGO}

160 Cahiers d'études japonaises nº 24

Mithout Anne-Lise, 2018, «'Récupérer le sexe qui m'a été volé' : la sexualité comme affirmation de soi chez Asaka Yuhô », Genre, sexualité et société, 19. (DOI : $10.4000 /$ gss.4200)

Oliver Michael, 1990, The Politics of Disablement, Palgrave Macmillan, London, $152 \mathrm{p}$.

Shakespeare Tom, 2006, "The Social Model of Disability”, in Davis Lennard (dir.), The Disability Studies Reader, Routledge, London, p. 214-221.

Stevens Carolyn, 2013, Disability in Japan, Routledge, London, 208 p.

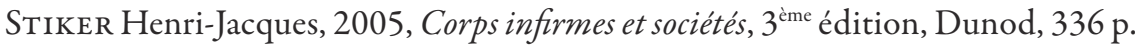

SugINo Akihiro 杉野昭博, 2007, Shögaigaku : rironkeisei to shatei 障害学: 理 論形成と射程 (Études sur le handicap : forme théorique et portée), Tōkyō daigaku shuppankai 東京大学出版会, Tōkyō, 294 p.

Suzuki Masako 鈴木雅子, 2010, Bungaku ni miru shōgaisha zō Yokozuka Kōichi cho 《hahayo! korosuna 》 文学にみる障害者像横塚晃一著『母よ! 殺 すな』(Les figures de personnes handicapées dans la littérature : « Maman! Ne me tue pas » de Yokozuka Kōichi), Nōmaraizēshon - shōgaisha fukushi ) ーマライゼーションー障害者福祉 (Normalisation - protection sociale des personnes handicapées), janvier. (URL : http://www.dinf.ne.jp/doc/ japanese/prdl/jsrd/norma/n342/n342020.html, consulté le 3 mai 2016)

Thomann Bernard, 2015, La Naissance de l'État social japonais : biopolitique, travail et citoyenneté dans le Japon impérial (1868-1945), Presses de Sciences Po, Paris, 450 p.

Toshimitsu Keiko 利光惠子, 2016, Sengo Nihon ni okeru josei shōgaisha e no kyōseiteki na funin shujutsu戦後日本における女性障害者への強制的な 不妊手術 (Les opérations de stérilisations forcées sur les femmes handicapées 
dans le Japon d'après-guerre), Ritsumeikan daigaku seizongaku kenkyū sentā 立命館大学生存学研究センター, Kyōto, 130 p.

YAMAZaKi Makoto 山崎亮, 2010, Yokozuka Kōichi no shisō to shūkyō - 1970 nendai 'Aoi shiba no kai' undōo megutte 横塚晃一の思想と「宗教」- 1

970 年代「青い芝の会」の運動をめぐって (La pensée de Yokozuka Kōichi et la religion - Autour du mouvement Aoi shiba no kai dans les années 1970), Shimane daigaku fukushi ronshū 島根大学社会福祉論集, 3, 3 mars, p. 54-68.

Yokozuka Kōichi 横塚晃一, 2007, Haha yo ! Korosu na 母よ！殺すな (Maman! Ne me tue pas), Seikatsu shoin 生活書院, Tōkyō, 464 p.

YORITAKa Tsunenobu 頼尊恒信, 2012, Nihon ni okeru shōgaigaku no genryū to shite no Aoi shiba no kai 日本における障害学の源流としての青い 芝の会 (Aoi shiba no kai : source des études sur le handicap au Japon), in Hori Masatsugu 堀正嗣 (dir.), Kyōsei no shōgaigaku - haijo to kakuri o koete 共生の障害学 排除と隔離を超えて (Les études sur le handicap fondées sur le vivre-ensemble : dépasser l'exclusion et l'isolement), Akashi shoten 明石書店, p. 48-77.

Résumé : Au début des années 1970, émerge pour la première fois au Japon un mouvement de personnes handicapées militant pour leur reconnaissance sociale et le respect de leurs droits humains. Ce mouvement, porté par l'association Aoi shiba no kai, naît de la critique de la relative tolérance pour les infanticides d'enfants handicapés et se fédère autour de la dénonciation de «l'eugénisme », un concept aux contours parfois flous, utilisé pour décrire un ensemble de situations très large. Cet article s'appuie sur les écrits de Yokozuka Kōichi, l'un des principaux leaders d'Aoi shiba no kai. Il vise à analyser comment, et avec quelles conséquences, Yokozuka Kōichi fait de la lutte contre « l'eugénisme » un instrument d'émancipation et d'affirmation de soi des personnes handicapées. On met ainsi en lumière le passage de la revendication d'un droit à l'existence des 
personnes handicapées à celle d'un droit à la reconnaissance du handicap en tant qu'identité particulière.

Mots-clés : eugénisme, handicap, Japon, militantisme, infanticide

In the early 1970s, the first Disability Rights movement appeared in Japan, campaigning for disabled people's social recognition and demanding respect for their human rights. This movement, led by the group Aoi shiba no kai, was highly critical of the widespread tolerance for infanticides of disabled children, and united in their denunciation of "engenics", a somehow blurred concept used to describe a very broad set of situations. This paper is based on Aoi shiba no kai leader Yokozuka Köichi's writings. It aims at analyzing how Yokozuka Köichi turned the struggle against "eugenics" into a tool for disabled people's emancipation and self-assertion. It highlights how the Disability Rights movement shifted from claiming a mere "right to existence" to asserting disability as a specific identity.

Keywords: eugenics, disability, Japan, activism, infanticide

キーワード：優生学、障害、日本、運動、子殺し 